Instituto Internacional de Investigación y Desarrollo Tecnológico Educativo INDTEC, C.A.

DOI: https://doi.org/10.29394/Scientific.issn.2542-2987.2020.5.17.16.292-310

OAI-PMH: http://www.indteca.com/ojs/index.php/Revista Scientific/oai

Ensayo Original / Original Essay

\title{
Educación con Tecnología en una Pandemia: Breve Análisis
}

\author{
Autoras: María del Carmen Crespo Argudo \\ Universidad Católica de Cuenca, UCACUE \\ merynena.ca@hotmail.com \\ Azogues, Ecuador \\ https://orcid.org/0000-0003-3419-7537 \\ María Cecilia Palaguachi Tenecela \\ Universidad Católica de Cuenca, UCACUE \\ chechism1@gmail.com \\ Azogues, Ecuador \\ https://orcid.org/0000-0003-1227-2102
}

\section{Resumen}

El mundo día a día se beneficia de la tecnología que trae una serie de ventajas, sobre todo en el ámbito educativo donde ha revolucionado la forma de enseñar, así como de aprender, hoy más que nunca necesitamos de ella para acceder a la educación en tiempos de pandemia, donde los estudiantes se ven impedidos para acudir a los establecimientos educativos debido a la cuarentena, esto hace que tanto estudiantes como docentes busquen formas de superar las brechas digitales para así poder acceder a una educación de calidad y calidez, es por eso que este ensayo le da mucho valor al docente que a pesar de ser inmigrante tecnológico se capacita para convertir la tecnología en una verdadera herramienta al servicio de la educación, para ello se presentan algunos ideas de cómo utilizar de la mejor manera los medios digitales para superar barreras que impidan el aprendizaje de los estudiantes y la correcta enseñanza por parte de los docentes.

Palabras clave: educación; tecnología de la información; informática educativa; digitalización.

Cómo citar este ensayo:

Crespo, M., \& Palaguachi, M. (2020). Educación con Tecnología en una Pandemia: Breve Análisis. Revista Scientific, 5(17), 292-310, e-ISSN: 2542-2987. Recuperado de: https://doi.org/10.29394/Scientific.issn.2542-2987.2020.5.17.16.292-310

Fecha de Recepción: 11-03-2020
Fecha de Aceptación: 18-06-2020
Fecha de Publicación: 05-08-2020 
Instituto Internacional de Investigación y Desarrollo Tecnológico Educativo INDTEC, C.A.

DOI: https://doi.org/10.29394/Scientific.issn.2542-2987.2020.5.17.16.292-310

OAI-PMH: http://www.indteca.com/ojs/index.php/Revista_Scientific/oai

Ensayo Original / Original Essay

\title{
Education with Technology in a Pandemic: Brief Analysis
}

\begin{abstract}
The world day by day benefits from technology that brings a series of advantages, especially in the educational field where it has revolutionized the way of teaching, as well as learning, today more than ever we need it to access education in times of a pandemic, where students are prevented from going to educational establishments due to quarantine, this makes both students and teachers look for ways to overcome digital gaps in order to access quality and warm education, that is why This essay gives a lot of value to the teacher who, despite being a technological immigrant, is trained to turn technology into a true tool at the service of education. For this, some ideas are presented on how to best use digital media to overcome barriers. that impede student learning and correct teaching by teachers.
\end{abstract}

Keywords: education; information technology; educational computing; digitization.

How to cite this essay:

Crespo, M., \& Palaguachi, M. (2020). Education with Technology in a Pandemic: Brief Analysis. Revista Scientific, 5(17), 292-310, e-ISSN: 2542-2987. Recovered from: https://doi.org/10.29394/Scientific.issn.2542-2987.2020.5.17.16.292-310

Date Received: 11-03-2020
Date Acceptance:

18-06-2020
Date Publication:

05-08-2020 
Instituto Internacional de Investigación y Desarrollo Tecnológico Educativo INDTEC, C.A.

DOI: https://doi.org/10.29394/Scientific.issn.2542-2987.2020.5.17.16.292-310

OAI-PMH: http://www.indteca.com/ojs/index.php/Revista_Scientific/oai

Ensayo Original / Original Essay

\section{Introducción}

La educación ha cambiado la forma de ser impartida debido al coronavirus, ahora el hogar se ha convertido en el único espacio posible para realizar esta actividad y así evitar los riesgos de un contagio en esta pandemia, este desafío solo es posible gracias a la tecnología que nos ofrece el mundo actual, que sin duda trae desventajas pues no todos tienen acceso a la red y aparatos tecnológicos, lo que ocasiona que los sectores vulnerables se queden atrás en sus aprendizajes acrecentando la brecha digital, pero la educación debe continuar ya sea con clases virtuales, con programas en los medios de comunicación convencionales, más la colaboración de los líderes en las comunidades rurales, las personas que pueden acceder a la tecnología estudiantes y maestros deben aprovechar al máximo los recursos disponibles sin olvidar el enorme sacrificio que hacen los que viven en condiciones de inequidad para alcanzar la educación.

Las Tecnologías de la Información y Comunicación (TIC), ha llegado a las aulas de educación a una gran velocidad y hoy exigen utilizarlos, pues la era digital ha sufrido muchas transformaciones tanto a nivel tecnológico y educativo, ha cambiado modelos pedagógicos haciendo que los maestros cambien las metodologías de trabajo y utilicen las tecnologías para mejorar los procesos de educación con recursos innovadores. Para De Moya y Cózar (2013), citados por Roblizo y Cózar (2015): las (TIC) en los últimos años del siglo XXI se han convertido en un impactante y revolucionario fenómeno social, presente en las acciones humanas, sean estas de trabajo, formación, consumo y diversión convirtiendo todo en un ambiente virtual en constante cambio.

La tecnología ha contribuido con beneficios incalculables a la humanidad, lo que se nota particularmente en la actualidad en la que el mundo enfrenta la pandemia del COVID-19; una de las ventajas es acortar distancias, para la comunicación con todos los habitantes de forma simultánea y al mismo tiempo sin que tengamos que establecer un contacto físico que ponga en 
Instituto Internacional de Investigación y Desarrollo Tecnológico Educativo INDTEC, C.A.

DOI: https://doi.org/10.29394/Scientific.issn.2542-2987.2020.5.17.16.292-310

OAI-PMH: http://www.indteca.com/ojs/index.php/Revista_Scientific/oai

Ensayo Original / Original Essay

riesgo de contagio. Para los autores Ramos (2014); y Rueda (2012), citados por Quintero-Corzo, Munévar-Molina y Munévar-Quintero (2015): las personas a través de computadoras, se relacionan con los demás en la "cibersociedad", en un espacio virtual donde no hay presencia física.

Gracias a las TIC los docentes pueden cumplir con el teletrabajo que les ha sido asignado debido a la emergencia sanitaria y se han visto forzados a dejar los tradicionalismos y poner en práctica nuevas formas de aprendizaje, que como indica García (2020): es mediante la educación a distancia conectados en línea, lo que ha cambiado la forma de educar; esta educación a distancia de probada calidad y eficacia, ha creado una multitud de modelos organizativos, tecnológicos y pedagógicos para una enseñanza y aprendizaje en base a una comunicación y colaboración ubicuas, instantáneas, y sostenidas en tiempo real, lo que en tiempos antiguos era impensable ya que solo era válida la educación presencial donde se utilizaban textos físicos memorísticos.

El propósito de este ensayo es analizar cómo está la educación en este tiempo de pandemia, así como hacer recomendaciones que permitan realizar la labor docente de la mejor manera posible, con la única meta que es buscar la calidad de la educación con ayuda de la tecnología.

\section{Desarrollo}

\subsection{Educando en la Pandemia}

La Organización Mundial de la Salud (OMS), a los 11 días del mes de marzo del año 2020, declara que el coronavirus COVID-19 pasa de ser una epidemia a ser una pandemia, por lo que las principales autoridades de cada Nación en el Mundo entero, han tomado medidas para controlar de cierta manera el colapso del sistema sanitario de cada país. Entre las acciones del Gobierno ecuatoriano está la declaración del estado de emergencia el 12 de marzo del 2020 por parte del Ministerio de Salud Pública del Ecuador, mientras 
Instituto Internacional de Investigación y Desarrollo Tecnológico Educativo INDTEC, C.A.

DOI: https://doi.org/10.29394/Scientific.issn.2542-2987.2020.5.17.16.292-310

OAI-PMH: http://www.indteca.com/ojs/index.php/Revista_Scientific/oai

Ensayo Original / Original Essay

que el Ministerio de Educación suspende las clases presenciales en todas las instituciones educativas y centros de desarrollo integral para la primera infancia, sin suspender las labores administrativas, docentes mediante el teletrabajo para reforzar y enseñar contenidos en los educandos de forma virtual; de esta forma se cambió en segundos todo el modelo educativo al que la comunidad estaba acostumbrada. El Ecuador ha sido un país que ha pasado por la suspensión de actividades académicas por paros, situaciones de riesgo como sismos, pero jamás ha experimentado el teletrabajo como medio para continuar con la educación en todo el país.

Señalando a Quintana (2019): el teletrabajo es una actividad que se realiza desde cualquier lugar gracias al uso de la tecnología. Esta modalidad de trabajo no es nueva, para Sánchez y Montenegro (2019): los avances en el conocimiento, así como la globalización económica hacen que las empresas ecuatorianas obtén por este sistema; entonces vale la pena decir que este es un beneficio prestado por la tecnología para la educación.

Existen muchos avances tecnológicos, por un lado, se tiene la digitalización de las cosas, donde toda la información puede estar disponible en la web, para acceso de usuarios desde cualquier dispositivo inteligente que esté interconectado; por otro lado la realidad aumentada que permite hacer visitas virtuales de lugares específicos para conocer sus características de forma tan real gracias a las herramientas 3D, para aprender de una manera activa al participar, indagar y experimentar dentro del mundo digital. Asimismo la automatización de procesos gracias al Internet de las cosas (IoT), permite que todos los servicios estén disponibles en la red, lo que genera datos valederos para acciones acertadas en temas no solo de educación, también de salud, ingeniería, desarrollo científico, entre otros, es decir a través de sensores que puedan generar datos para ser procesados y almacenados como experiencias, para el desarrollo incluso de la inteligencia artificial obteniendo las mejores respuestas para la solución de problemas. 
Además, la industria 4.0 es el nuevo tipo de trabajo que reemplaza en gran parte la labor humana, incluso para actividades difíciles como el rescate de personas atrapadas en terremotos, o para experimentos con químicos peligrosos y altas radiaciones gracias a nano robots.

En los marcos de las observaciones anteriores las formas de enseñar también han evolucionado, gracias a las TIC y los paradigmas han ido adoptando nuevas estrategias, como la innovación en la pedagogía, por medio de las (TIC) y las Tecnologías para el Aprendizaje y Conocimiento (TAC) ya que los estudiantes viven en la sociedad red, donde ocurren gran parte de los aprendizajes, esto durante la pandemia a resultado un oasis para estudiantes y docentes que desean seguir aprendiendo, donde se aprovecha las múltiples ventajas de la tecnología para formar a los cyber-estudiantes, donde el aula (espacio físico) es un mito, ya que la educación virtualizada es el auge y éxito de los centros educativos, basados en experimentos y simulaciones, gracias a las herramientas tecnológicas desarrolladas, para organizar contenidos, evaluaciones y realizar control de tareas.

Es necesario estar preparados para enfrentar los cambios que exigen las nuevas modalidades de aprendizaje, así como el poner en práctica los conocimientos sobre las nuevas tecnologías (TIC), para Pinedo (2017): el acceso a una educación de calidad, es dotar al docente de herramientas que permitan complementar al contenido curricular con las tecnologías y herramientas disponibles, las cuales son un apoyo para poder presentar una clase diferente, creativa, dinámica, participativa, constructivista, e inclusivo para las estudiantes con discapacidad, de esta forma se despierta el interés de los participantes por aprender; así pues, es importante capacitar a los docentes en (TAC) para que cada educador pueda utilizar diferentes programas en sus clases, para hacerlo de forma interactiva; y podrá configurar el aula virtual de la especialidad que imparte con el objetivo de estar acorde con la tecnología. 
Como dicen Cueva, García y Martínez (2019): la formación educativa de los estudiantes permite el uso adecuado de las TIC y mejora los resultados educativos, por lo que es fundamental la capacitación docente, en donde algunos están conscientes de que no están preparados para vivir en el mundo de la web 2.0, ya que no recibieron los conocimientos necesarios en su formación docente, pero buscan capacitarse para poder educar a los nativos tecnológicos y no quedarse atrás de ellos.

Es necesario diferenciar los grupos de personas que utilizan tecnologías, los nativos e inmigrantes digitales, acorde con Prensky (2010): los primeros son los que nacieron en un mundo tecnológico utilizando la "lengua digital", mientras en el segundo caso no, pero tratan de adaptarse al entorno y al ambiente, aunque conservando la conexión con el pasado. Los llamados nativos digitales no le temen a la tecnología, les gusta y cada día siguen aprendiendo por intuición mediante el ensayo error, dejando a sus docentes en desventaja generando sentimientos de incapacidad.

De acuerdo con, Alva (2015a): uno de los retos a superar es las barreras de uso, que es la forma en la que las personas aprovechan al máximo las TIC y las ponen en práctica según las necesidades; hay cantidad de personas sobre todo los inmigrantes digitales que tienen dificultades para acceder a estas nuevas tecnologías trayendo por consiguiente la pobreza digital que se conceptualiza como la carencia en el uso y el acceso de las (TIC)

En conformidad con Martínez (2018a): la ausencia de laboratorio de cómputo, herramientas tecnológicas, red, mantenimiento, falta de recursos económicos y cursos de formación en cuanto a los usos de la tecnología y equipos informáticos, preocupa a los maestros que hacen notar la inexperiencia al momento de manejar la tecnología, lo que genera sentimientos de inseguridad y estrés, pero también, que tienen un nivel de resistencia al cambio; dependiendo de la edad en que se encuentren, provocando en sus estudiantes una educación pobre en recursos. 
Instituto Internacional de Investigación y Desarrollo Tecnológico Educativo INDTEC, C.A.

DOI: https://doi.org/10.29394/Scientific.issn.2542-2987.2020.5.17.16.292-310

OAI-PMH: http://www.indteca.com/ojs/index.php/Revista_Scientific/oai

Ensayo Original / Original Essay

La mejor inversión que el gobierno puede hacer en educación, es en la formación de los docentes inmigrantes digitales, ya que la educación contribuye a la equidad de un País. Por otra parte, si los docentes están preparados y tienen acceso a herramientas digitales, la escuela permite que todos los estudiantes estén relacionados con la tecnología y puedan manejarla, como un medio de gran apoyo para la población estudiantil de escasos recursos económicos y no estén en desventaja con aquellos que poseen y manejan tecnología en sus hogares.

Hoy el coronavirus obliga a todos los docentes a utilizar la tecnología; pero el reto es aún más grande, no implica solo el uso de las herramientas tecnológicas, sino que se debe educar por medio de ellas a los estudiantes, es aterrador imaginar a los docentes que no usaban para nada las TIC en las aulas, adaptarse a un ambiente virtual, puede causar miedo, como ha todo lo desconocido. A juzgar por Marín (2019): la formación a distancia debe estar centrada en el estudiante y contar con las adecuaciones curriculares en función de sus necesidades, esto es que el docente debe capacitarse, pero también dejar el miedo a un lado y explorar para aprender mediante el ensayo error el manejo de aparatos tecnológicos, ya que para Martínez (2018b): las herramientas TIC ayudan y revolucionan los procesos educativos.

Es muy importante contar con las herramientas básicas dentro de las Instituciones Educativas para poder aprovechar la tecnología y permitir que los estudiantes se beneficien de ella. En cuanto a eso, Navarrete, Vera y Idrovo (2019): exponen que el Gobierno ecuatoriano debe replantear sus estrategias para cumplir con los estándares de calidad educativa, como un currículo compacto para lograr la excelencia académica con capacitaciones a los docentes, el acceso a internet en todas las Instituciones Educativas, la creación de plataformas virtuales interactivas y la búsqueda de directores con perfil académico idóneo, de acuerdo a las demandas actuales, para que lideren, supervisen la investigación y evaluación. 
Instituto Internacional de Investigación y Desarrollo Tecnológico Educativo INDTEC, C.A.

DOI: https://doi.org/10.29394/Scientific.issn.2542-2987.2020.5.17.16.292-310

OAI-PMH: http://www.indteca.com/ojs/index.php/Revista_Scientific/oai

Ensayo Original / Original Essay

En referencia a las consideraciones anteriores, el docente es la clave educativa en esta pandemia, por ello es fundamental entender que las TIC son primordiales para la innovación educativa, pero si no van acompañadas de una buena planificación pedagógica, así como de estrategias y recursos innovadores sería un desperdicio de tecnología; es decir se educa para hoy con metodologías de ayer, esperando que los estudiantes rindan en el mañana. Para lograr un cambio real en la educación los docentes deben estar preparados para desaprender, dejando atrás sus tradicionalismos, buscando mejorar su capacidad crítica y reflexiva para producir cambios en los educandos a través de su experiencia.

Complementa Delauro (2011): que los docentes no solo deben tener competencias tecnológicas gracias a la capacitación o formación en esta área, sino necesitan actitud para poder alcanzar estas competencias con procesos de capacitación progresivos; estas competencias pueden ser: manejar las operaciones básicas de las computadoras, editores de textos, programas de comunicación, uso de plataformas educativas para estar en contacto con la comunidad estudiantil, editores gráficos, diferentes navegadores y buscadores de internet, utilizar programas para presentaciones de clase, plantillas de cálculo, base de datos y utilizar programas especializados en la asignatura que imparten.

Para innovar e implementar proyectos educativos basados en las TIC, es necesario eliminar la barreras o brechas digitales, por otro lado, Alva (2015b): manifiesta que existen dimensiones que se son muy importantes en la brecha digital: la primera es la dimensión económica en donde se debe considerar los precios de las TIC ya que hay muchas personas que no tienen para pagar aparatos tecnológicos, ni internet; la segunda es la dimensión política porque es necesario tomar en cuenta las regulaciones en cuanto a las TIC; la tercera es la dimensión tecnológica que hace referencia a los recursos para el uso de las TIC ya que en muchos lugares las personas no 
Instituto Internacional de Investigación y Desarrollo Tecnológico Educativo INDTEC, C.A.

DOI: https://doi.org/10.29394/Scientific.issn.2542-2987.2020.5.17.16.292-310

OAI-PMH: http://www.indteca.com/ojs/index.php/Revista_Scientific/oai

Ensayo Original / Original Essay

pueden acceder a este servicio por ser muy lejanas a la ciudad y a las redes de conexión; la quinta es la dimensión social y tiene que ver con la inequidad social de la población que no van a poder adquirir estas nuevas tecnologías y la última es la dimensión cultural que tiene que ver con los pensamientos y la actitud hacia las tecnologías.

La educación debe ser integradora, no es suficiente la preocupación por los contenidos, debe actuar de acuerdo a la realidad buscando desarrollar al ser humano, para ello es imprescindible que tenga la capacidad de evolucionar con el tiempo y escalar a los nuevos escenarios en los que la sociedad se desenvuelve, por lo que, las capacitaciones son muy importantes con contenidos realmente necesarios, como redes de comunicación y aprendizaje para generar espacios de trabajo colaborativo para el intercambio de experiencias, realizar tutoriales, blogs, grupos de chat en las redes sociales para solventar dudas, videoconferencias, plataformas educativas y más; generando trabajos autónomos que contribuyan al equipo y en especial para que los estudiantes aprendan a realizar trabajo colaborativo.

Las aplicaciones de la web 2.0 más usadas en esta crisis sanitaria son las redes sociales, como medio de comunicación entre representantes legales y representados, ya que por su versatilidad también ayudan a generar conocimientos, entre las más utilizadas están: Facebook, Twitter, Linkedln, Instagram, Likee y TikTok, entre otras.

Gracias a estas herramientas se pueden desarrollar actividades dinámicas e interactivas que faciliten y fortalezcan los procesos educativos, mediante la creación de grupos para preguntar sobre las tareas que no han sido entendidas, buscando la colaboración de sus pares, tutoría docente para fortalecer aprendizajes; a través de estas plataformas digitales se puede subir videos, fotos, lo que permite interactuar de forma divertida para generar aprendizaje y colaborar con el resto a pesar de no estar presentes físicamente; los docentes también recurren a estos medios para realizar reuniones de 
trabajo, asignar tareas, resolver inquietudes, generar aprendizajes, hacer video llamadas, con la ayuda de otras aplicaciones pueden realizar tutoriales, compartir información e imágenes que ayudan a la construcción participativa de conocimientos en los estudiantes.

Las redes sociales han aparecido como un medio de comunicación, pues en la antigüedad uno de los medios que empleaban las personas para comunicarse eran cartas, y estas han ido evolucionado hasta convertirse en la actualidad datos que se encuentran en una base de datos, alojados en servidores físicos y en la nube, que tienen un gran impacto en la era moderna facilitando la comunicación de los aprendizajes de forma virtual y entretenida; sin embargo al estar rodeados de abundante información, también existen riegos de desinformación, por eso es necesario saber discernir la información científica y la generada a través de experiencias u opiniones, ya que esto perjudica principalmente a niños y jóvenes que todavía no tienen un criterio aceptable; así también, estas herramientas son tan interesantes que pueden crear adicciones por el abuso en su uso.

En el caso de los niños de educación inicial el acceso a redes sociales depende totalmente del representante legal, y se ha visto que la aplicación más utilizada es el WhatsApp por el fácil manejo y acceso para comunicarse; conviene subrayar que la docente genera grupos, para que los representantes descarguen el material: imágenes, audios, videos, archivos, promoviendo el aprendizaje de los más pequeños y manteniendo informados a los representantes legales con directrices actualizadas del Ministerio de Educación, para conseguir el desarrollo de las destrezas que se plantean en el currículo con el trabajo en equipo, el docente debe diseñar y enviar actividades considerando la individualidad de los educandos, así mismo, es preciso tomar en cuenta que todas las familias tengan a su alcance los recursos necesarios para evitar que salgan de la cuarentena.

Añaden Garita-González (2018): que existen innovaciones que se 
Instituto Internacional de Investigación y Desarrollo Tecnológico Educativo INDTEC, C.A.

DOI: https://doi.org/10.29394/Scientific.issn.2542-2987.2020.5.17.16.292-310

OAI-PMH: http://www.indteca.com/ojs/index.php/Revista_Scientific/oai

Ensayo Original / Original Essay

utilizan en otros países y que vale la pena revisarlas para aplicarlas en las instituciones que cuenten con recursos, como es el caso de un evento universitario realizado en Finlandia, donde se describe un modelo pedagógico denominado Open Innovation Space, en la cual se enseña mediante la plataforma Moodle, y se hacen practicas mediante la simulación, se crean productos a partir de un software de diseño, en el que cuentan bibliotecas automatizadas, impresoras 3D, y por otra parte, se realizan videoconferencias mediante Zoom, Cisco Webex Meetings y servicios en la nube.

Otras plataformas fáciles de usar son: Edmodo, Microsoft Teams, Schoology, Ilias y Tiching, etc. Entra las más usadas por el Ministerio de Educación, tenemos: Educar Ecuador, MinEduc y más, que están al alcance de todos, solo se necesita poner en el buscador de preferencia lo que se desea encontrar para potenciar al máximo la educación y con un clic se encuentran tutoriales para el manejo de cualquier plataforma, lo indispensable para ello es una actitud positiva, deseos de investigar y la ausencia del miedo a explorar lo desconocido.

Para los pequeños de educación inicial, es primordial actividades divertidas, esto se puede realizar fácil y rápido con la ayuda de YouTube, donde se puede buscar, descargar, editar, subir y compartir videos, tutoriales, documentales, y eventos en vivo; de igual forma, existe la posibilidad de interactuar con los demás; todas estas características hacen de este sitio web sea el más usado después de Facebook.

Las herramientas tecnológicas nos dan muchos beneficios pero hay que saber explotarlas al máximo como es el caso de los programas de Ofimática de Microsoft que a menudo se usan: Word, Excel, PowerPoint y Access, ya que son muy conocidos y pensamos equivocadamente que las dominamos, un ejemplo de esto es PowerPoint, cuantos docentes lo utilizan para hacer presentaciones monótonas sin saber que esta herramienta puede ser una opción para trabajar a distancia, mejorando los procesos de aprendizaje con 
Instituto Internacional de Investigación y Desarrollo Tecnológico Educativo INDTEC, C.A.

DOI: https://doi.org/10.29394/Scientific.issn.2542-2987.2020.5.17.16.292-310

OAI-PMH: http://www.indteca.com/ojs/index.php/Revista_Scientific/oai

Ensayo Original / Original Essay

los estudiantes mediante la grabación de audio y video, que proporcionen comentarios o expongan la presentación, claro que no está disponible para las opciones gratis de office. Sin embargo, existe una forma de conseguir esta y otras ventajas a través de una de tantas aplicaciones gratuitas que puede obtenerse en línea o a través de su descarga e instalación en el ordenador.

Este aplicativo se llama Screencast-O-Matic, que ayuda a grabar todo lo que se desea mostrar de la pantalla del ordenador, también se puede grabar la cara del presentador con la webcam haciendo un video tutorial completo, lo mejor es que si no sabes cómo usarlo, puedes ver un video tutorial en YouTube para sacarle muchas ventajas a esta aplicación.

Cuando se regrese a las clases presenciales se puede aprovechar la famosa recuperación pedagógica para ver recursos llamativos, con medios tecnológicos para reforzar los aprendizajes, en el caso de educación inicial se podría implementar un ambiente de aprendizaje llamado tecnologías donde los niños puedan interactuar, explorar y aprender el uso de ciertos aparatos tecnológicos, quien no tiene un teléfono viejo en casa, o una computadora antigua que no la use pero todavía sirva, todos los docentes podrían donar aparatos tecnológicos y equipar este ambiente para facilitar el aprendizaje.

Los educandos que tienen posibilidades económicas pueden usar sus tablets, celulares o computadoras para descargar video juegos y aplicaciones móviles y dependiendo del programa que se descarguen puede beneficiarse en diferentes aspectos ya sea cognitivos, motrices, de lenguaje, social, mejorando procesos de aprendizaje. Cualquier programa interactivo o no en manos de un docente competente puede convertirse en una herramienta poderosa que desarrolla la capacidad multisensorial de los estudiantes para enfrentar los retos del mundo digital.

En la actualidad las TIC revolucionan los mercados y la educación, con los pizarrones interactivos digitales, por consiguiente, Calixto (2011): cree en los beneficios de su utilización y por tal motivo ha descrito los siguientes: Smart 
Board y eBeam, la primera con un proyector de presentaciones y juegos interactivos, son una de las experiencias que les va a encantar a los estudiantes, para su utilización se puede usar la mano como un ratón, consiguiendo trazos suaves; la otra pizarra eBeam es fácil de transportar ya que es portátil; también pueden realizarse mediante una fabricación casera, utilizando una videoconsola "Wii" y un bolígrafo; esta es una de las posibilidades para utilizar en el ámbito educativo sin necesidad de contar con computadoras fijas que por el uso de los estudiantes pueden dañarse y necesitan manteamiento constante; los pizarrones interactivos digitales en las aulas son una de las maneras de innovar y de mejorar la calidad de educación.

\section{Conclusiones}

La tecnología es una herramienta para la educación que no puede faltar, permite la comunicación con toda la comunidad educativa, contribuye a la interacción entre docentes y estudiantes, permite aprendizajes significativos en los estudiantes y, de que los docentes necesitan de la tecnología incluso en el momento de planificar, caso contrario en las experiencias de aprendizaje los recursos empleados pueden ser pobres y perjudicar en vez de ayudar al aprendizaje de sus estudiantes.

Para educar con la tecnología es necesario que los profesionales docentes cambien de mentalidad y se atrevan a salir de la zona de confort para experimentar sin miedo la web 2.0, apoyados del acompañamiento pedagógico que debe brindar el Ministerio de Educación, así como las capacitaciones generadas por sus propios medios con el único fin de saber utilizar todas las herramientas tecnológicas que ayuden a la enseñanzaaprendizaje, fundamentalmente en momentos difíciles como los que hoy está atravesando el mundo por la pandemia.

En base a un levantamiento de la infraestructura tecnológica con la que cuenta cada institución educativa, es necesario armar un Plan Estratégico de 
Tecnología y Sistemas de Información (PETI), para implementar una política de uso progresivo de las TICS en el aula, con normativas, reglas de acceso y fuentes de información científicas para que la información que llegue sea válida y no solo un montón de información basura; asimismo para que en este proyecto se detallen instrumentos de evaluación basados en ciertas destrezas o estándares universales de rendimiento.

Buscar herramientas gratuitas offline que puedan ser utilizadas en la institución, ya sean en teléfonos inteligentes, tablets, computadoras con aplicaciones para redacción, cálculo, edición, búsqueda y control de actividades; igualmente para el ahorro de recursos económicos ya que dichos simuladores evitarían la compra de componentes o equipos electrónicos físicos con precios considerables.

Generar un modelo de educación activa con la ayuda tecnológica, con pizarras interactivas digitales que ayuden al estudiante a que aprenda mientras se divierte, para que se pueda interactuar con diversos contenidos mediante materiales gráficos y audiovisuales que generen motivación al aprender, además existen aplicativos que permiten elaborar en las diferentes asignaturas, pruebas estructuradas, visitas virtuales y simulaciones para desarrollar en el estudiante experiencias en base al método ensayo-error, lo que permite la resolución de problemas y la generación de habilidades para el siglo XXI.

\section{Referencias}

Alva, A. (2015a,b). Los nuevos rostros de la desigualdad en el siglo XXI: la brecha digital. Revista Mexicana de Ciencias Políticas y Sociales, 60(223), 265-286, e-ISSN: 0185-1918. Recuperado de:

http://dx.doi.org/10.1016/S0185-1918(15)72138-0

Calixto, M. (2011). E-learning: pizarras y tabletas digitales interactivas, videoconferencias, vídeos docentes, entornos colaborativos y 
Instituto Internacional de Investigación y Desarrollo Tecnológico Educativo INDTEC, C.A.

DOI: https://doi.org/10.29394/Scientific.issn.2542-2987.2020.5.17.16.292-310

OAI-PMH: http://www.indteca.com/ojs/index.php/Revista_Scientific/oai

\section{Ensayo Original / Original Essay}

plataformas para la docencia online. ISBN: 978-84-694-5333-9.

Cartagena, España: Universidad Politécnica de Cartagena. Recuperado de: http://hdl.handle.net/10317/2302

Cueva, J.., García, A., \& Martínez, O. (2019). El conectivismo y las TIC: Un paradigma que impacta el proceso enseñanza aprendizaje. Revista Scientific, 4(14), 205-227, e-ISSN: 2542-2987. Recuperado de: https://doi.org/10.29394/Scientific.issn.2542-2987.2019.4.14.10.205$\underline{227}$

Delauro, M. (2011). Unidad 1-a: Nuevos escenarios, nuevos roles docentes, nuevas competencias. La tutoría en ambientes virtuales de aprendizaje. Washington, D.C., Estados Unidos: Instituto de Formación Docente, Virtual Educa.

García, L. (2020). Bosque semántico: ¿educación/enseñanza/aprendizaje a distancia, virtual, en línea, digital, eLearning...?. RIED: Revista Iberoamericana de Educación a Distancia, 23(1), 9-28, e-ISSN: 13903306. Recuperado de: http://dx.doi.org/10.5944/ried.23.1.25495

Garita-González, I. (2018). Aprendiendo para el futuro: una experiencia en Finlandia. Revista Espiga, 17(35), 106-115, e-ISSN: 2215-454X. Recuperado de: $\underline{\text { http://dx.doi.org/10.22458/re.v17i35.2093 }}$

Martínez, O. (2018a,b). Uso de las Tecnologías de la Información y la Comunicación en la Educación Básica. Revista Scientific, 3(10), 154174, e-ISSN: 2542-2987. Recuperado de:

https://doi.org/10.29394/Scientific.issn.2542-2987.2018.3.10.8.154-174 Marín, N. (2019). Las Tecnologías de Información y Comunicación: Una Gestión Educativa desde la Plataforma Moodle. Revista Scientific, 4(12), 329-339, e-ISSN: 2542-2987. Recuperado de:

https://doi.org/10.29394/Scientific.issn.2542-2987.2019.4.12.17.329339

Navarrete, G., Vera, M., \& Idrovo, K. (2019). Un nuevo mundo educativo: 
Instituto Internacional de Investigación y Desarrollo Tecnológico Educativo INDTEC, C.A.

DOI: https://doi.org/10.29394/Scientific.issn.2542-2987.2020.5.17.16.292-310

OAI-PMH: http://www.indteca.com/ojs/index.php/Revista_Scientific/oai

Ensayo Original / Original Essay

organización, funcionamiento y estructura. Una propuesta a la educación ecuatoriana. Espirales: revista multidisciplinaria de investigación, 3(26), 50-59, e-ISSN: 2550-6862. Recuperado de: https://doi.org/10.31876/re.v3i26.459

Pinedo, I. (2017). Programa de Capacitación Docente: Tecnologías del Aprendizaje y del Conocimiento - TACs. Trabajo de Grado. Panamá: Universidad Especializada de las Américas. Recuperado de: http://repositorio2.udelas.ac.pa/handle/123456789/32

Prensky, M. (2010). Nativos e Inmigrantes Digitales. Cuadernos SEK 2.0. San Sebastián de los Reyes, España: Distribuidora SEK, S.A.

Quintana, J. (2019). Estudio de avances en la implementación del teletrabajo en la Universidad Nacional Abierta y a Distancia UNAD 2016 - 2019. Tesis. Bogotá, Colombia: Universidad Nacional Abierta y a Distancia UNAD. Recuperado de:

https://repository.unad.edu.co/handle/10596/31117

Quintero-Corzo, J., Munévar-Molina, R., \& Munévar-Quintero, F. (2015).

Nuevas tecnologías, nuevas enfermedades en los entornos educativos. Hacia la promoción de la salud, 20(2), 13-26, e-ISSN: 0121-7577. Recuperado de:

https://www.redalyc.org/articulo.oa?id=309143500002

Roblizo, M., \& Cózar, R. (2015). Usos y competencias en TIC en los futuros maestros de educación infantil y primaria: hacia una alfabetización real para docentes. Píxel-Bit: Revista de Medios y Educación, (47), 2339, e-ISSN: 2171-7966. Recuperado de:

https://recyt.fecyt.es/index.php/pixel/article/view/61669

Sánchez, G., \& Montenegro, A. (2019). Teletrabajo una propuesta de innovación en productividad empresarial. 593 Digital Publisher CEIT, 4(5-1), e-ISSN: 2588-0705. Recuperado de: https://doi.org/10.33386/593dp.2019.5-1.133 


\section{María del Carmen Crespo Argudo \\ e-mail: merynena.ca@hotmail.com}

Nacida, en Azogues, provincia del Cañar, Ecuador, el 17 de

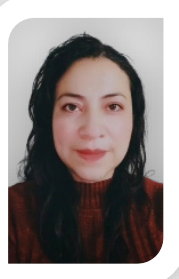
enero del año 1987. Licenciada en Tecnologías de Estimulación Temprana en Salud por la Universidad de Cuenca (UCUENCA); promotora de niños de cero a cinco años en los Centros de Desarrollo Infantil del Municipal Azogues, Docente de educación inicial en la Unidad Educativa "Nicanor Aguilar Maldonado"; Docente de educación Inicial en la Institución Educativa "Alonso Torres". 
Instituto Internacional de Investigación y Desarrollo Tecnológico Educativo INDTEC, C.A.

DOI: https://doi.org/10.29394/Scientific.issn.2542-2987.2020.5.17.16.292-310

OAI-PMH: http://www.indteca.com/ojs/index.php/Revista_Scientific/oai

Ensayo Original / Original Essay

\section{María Cecilia Palaguachi Tenecela}

e-mail: chechism1@gmail.com

Nacida en Cañar, Ecuador, el 28 abril del año 1992. Realice

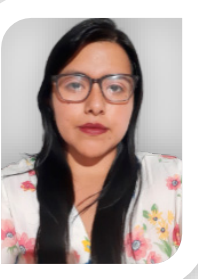
mis estudios Universitarios en el área de Educación Inicial y Parvularia por la Universidad Católica de Cuenca (UCACUE); y en el momento trabajo con niños pequeños de educación inicial de 3 a 5 años en el CEI "Alonso Torres" de la ciudad de Azogues.

El contenido de este manuscrito se difunde bajo una Licencia de Creative Commons ReconocimientoNoComercial-Compartirlgual 4.0 Internacional 